SHORT REPORT

\title{
Oral isotretinoin in the treatment of recalcitrant condylomata acuminata of the cervix: a randomised placebo controlled trial
}

\author{
S Georgala, A C Katoulis, C Georgala, E Bozi, A Mortakis
}

Sex Transm Infect 2004;80:216-218. doi: 10.1136/sti.2003.006841

Background: Conventional therapies for human papillomavirus (HPV) infection are often associated with unsatisfactory response rates and high recurrence rates. The use of a systemic agent may more effectively control the virus.

Objectives: To investigate the efficacy and safety of low dose oral isotretinoin in recalcitrant condylomata acuminata (RCA) of the cervix.

Methods: Double blind placebo controlled clinical trial. 60 women, aged 21-43 years, with RCA of the cervix, refractory to at least one conventional therapy, were randomly assigned to receive either isotretinoin, $0.5 \mathrm{mg} / \mathrm{kg}$ daily for 12 weeks (group 1), or placebo (group 2).

Results: Of the 28 evaluable group 1 patients, nine (32.1\%) responded to the treatment completely, 11 (39.2\%) responded partially, and eight $(28.5 \%)$ did not respond. Of the 25 group 2 patients, no one responded to the treatment completely, two (8\%) responded partially, and $23(92 \%)$ did not respond. The therapeutic difference between patients receiving active and placebo therapy was statistically significant $\left(\chi^{2}=19.35, p<0.001\right)$. Only one $(11.1 \%)$ of the complete responders experienced recurrence during the 12 month follow up. Side effects were generally mild and resolved upon completion of therapy.

Conclusions: Compared to placebo, low dose oral isotretinoin showed considerable efficacy with insignificant and reversible side effects and a low recurrence rate. Isotretinoin may represent an efficacious and safe alternative systemic form of therapy for RCA of the cervix.

\footnotetext{
$\Lambda$ nogenital human papillomavirus (HPV) infection is a multicentric process, manifested by condylomata acuminata and associated with latent disease. Genital HPV infection is important because of its high incidence and prevalence, but also because of its association with cervical cancer, the second most common malignancy in women worldwide and a leading cause of mortality among young women. ${ }^{1}$

In the absence of specific anti-HPV agents, conventional management for genital warts is often associated with unsatisfactory response rates and high recurrence rates. ${ }^{2}$ Since most applicable methods act locally, the use of a systemic agent may more effectively control the virus.

Retinoids are synthetic vitamin A derivatives, which act on target cells by binding and activating nuclear receptors that lead to the expression of genes regulating cell growth and differentiation. ${ }^{3}$ Retinoids have been shown to induce regression of cervical dysplasia, ${ }^{4}$ as well as to be effective against skin and cervical cancer. ${ }^{5}$
}

Our aim was to investigate the efficacy and safety of low dose oral isotretinoin in the treatment of recalcitrant condylomata acuminata (RCA) of the cervix.

\section{PATIENTS AND METHODS}

Sixty women with RCA entered this study at "A Sygros" Hospital, Athens Greece, from 1999 to 2002. The protocol was approved by the ethics committee of our hospital. Informed consent was obtained in each case.

The evaluation at entry included history taking, clinical examination, colposcopy, Papanicolaou smear, blood counts and biochemistry profile, and lesional biopsy. Eligibility criteria included a cytohistologically confirmed diagnosis of genital warts with at least 3 months' duration, lesions refractory to conventional therapy, age older than 18 years, negative human immunodeficiency virus (HIV) serology, no evidence of cervical dysplasia or malignant degeneration, no contraindications for isotretinoin administration-that is, no pregnancy in course and normal medullar, renal, hepatic, and lipidaemic laboratory parameters; no history of local or systemic anti-wart therapy, or cytotoxic, or immunomodulatory treatment within 4 weeks from entry.

The patients were randomised into two treatment groups of 30 subjects each, using simple randomisation. They were assigned to receive either oral isotretinoin (group 1), or placebo (group 2) given in an equivalent number of identical in appearance capsules, on a double blind basis. Oral isotretinoin (Roaccutane, Roche) dosage was $0.5 \mathrm{mg} / \mathrm{kg}$ daily for 12 weeks. The capsules were dispensed to the patients by a third party. No other topical or systemic medication was administered during the study period. Patients were advised against pregnancy and were instructed to practise an adequate form of contraception, as well as to use condoms in order to avoid re-infection.

Response was evaluated by recording the number of lesions and by bidimensional measurements of the lesions, mapped on a schematic diagram of the cervix. This study was performed at entry, every 4 weeks thereafter, and at the end of therapy. Clinical evaluation was blinded to the treatment. Complete response was defined as the total clearance of cervical lesions; partial response was considered as a reduction of lesions equal to or greater than $50 \%$; no response was considered to be less than $50 \%$ reduction in size. The reappearance of lesions after complete response was considered as relapse. Complete responders were followed up monthly for 12 months. Partial responders and non-responders were given other treatments.

Statistical analysis involved the $\chi^{2}$ test (Yates's correction included). The level of significance was fixed at $\alpha=5 \%$.

Abbreviations: HPV, human papillomavirus; RCA, recalcitrant condylomata acuminata 
Table 1 Patient flow chart and efficacy at 12 weeks for oral isotretinoin $0.5 \mathrm{mg} / \mathrm{kg} /$ day (group 1) versus placebo (group 2)

\begin{tabular}{lll}
\hline & Group 1 & Group 2 \\
\hline No of patients entered & 30 & 30 \\
No of patients withdrawn (side effects or poor compliance) & 2 & 2 \\
No of patients lost to follow up & 0 & 3 \\
No of evaluable patients & 28 & 25 \\
Response & $9(32.1 \%)$ & $0(0 \%)$ \\
$\quad$ Complete response & $11(39.2 \%)$ & $2(8 \%)$ \\
$\quad$ Partial response & $8(28.5 \%)$ & $23(92 \%)$ \\
$\quad$ No response & $13.1(5.4)$ & $12.8(4.7)$ \\
Mean area of the lesions $\left(\mathrm{mm}^{2}\right)$ & $6.9(3.8)$ & $11.9(4.4)$ \\
$\quad$ Before treatment (SD) & & \\
$\quad$ After treatment (SD) & & \\
\hline
\end{tabular}

Toxicity was monitored, on a monthly basis, both clinically and through laboratory tests, which included complete blood counts, renal and hepatic parameters, and lipidic profile. In case of significant toxicity treatment was discontinued.

\section{RESULTS}

Sixty women aged 21-43 years (mean 32.3 (SD 4.9) years) were enrolled. The duration of lesions varied from 721 months. Involvement of the external genitalia was found in $40 \%$ of the patients. Both groups were well matched for age, duration of infection, and extent of involvement.

Fifty three patients were evaluable for response and toxicity. Seven patients were withdrawn from the study because of poor compliance or side effects or were lost to follow up.

The primary end point for evaluation of efficacy was at 12 weeks. The results are summarised in table 1 . The therapeutic difference (percentage of responders) between patients receiving active and placebo therapy was statistically significant $\left(\chi^{2}=19.35, \mathrm{p}<0.001\right)$.

At the secondary endpoint for evaluation of efficacy (completion of 12 month follow up), out of nine complete responders eight remained in remission, whereas one (11.1\%) experienced a recurrence at week 43.

Isotretinoin was generally well tolerated. Side effects were of mild or moderate severity and resolved upon completion of therapy (table 2). One patient dropped out on week 10 because of persistently elevated serum transaminases, which returned to normal a few weeks after the treatment was stopped.

\section{DISCUSSION}

Our results seem to support the feasibility and effectiveness of systemic retinoids in the treatment of RCA. Considering the potential of genital warts to resolve spontaneously in up to $20 \%$ of the cases, it cannot be ruled out that some of the responses were actually spontaneous remissions. However, this possibility seems less likely since the lesions of our patients had been resistant to previous treatments and the

Table 2 Side effects of oral isotretinoin therapy in subjects with recalcitrant condylomata acuminata

\begin{tabular}{ll}
\hline Side effect & Frequency (\%) \\
\hline Cheilitis & 89.2 \\
Skin and mucosal dryness & 78.5 \\
Hair thinning/transient hair loss & 10.7 \\
Conjunctivitis & 7.1 \\
Elevation of triglycerides and/or cholesterol & 10.7 \\
Elevation of transaminases & 14.2 \\
\hline
\end{tabular}

effectiveness of isotretinoin was documented in comparison with placebo.

Previous experience with isotretinoin in genital warts is conflicting. Olsen et al compared systemic interferon alfa-nl and isotretinoin $(1 \mathrm{mg} / \mathrm{kg}$ for 6 weeks $){ }^{6}$ None of the eight patients treated with isotretinoin responded. Tsambaos et al treated 56 male patients with RCA using isotretinoin $1 \mathrm{mg} / \mathrm{kg}$ for 3 months. ${ }^{7}$ Of these, $39.6 \%$ responded completely and $13.2 \%$ responded partially. Two recurrences $(9.5 \%)$ occurred during the 1 year follow up. Cardamakis et al compared the combination of interferon $\alpha-2 \mathrm{a}$ and isotretinoin with isotretinoin alone ( $1 \mathrm{mg} / \mathrm{kg}$ for 3 months) in 57 women and in 87 men. $^{8}$ There was no statistical difference in remission rates, but the duration of treatment was significantly shorter and the recurrence rate was lower for the combination therapy.

The mechanism through which retinoids exert their beneficial effect in HPV infection remains unknown. It has been suggested that retinoids may have some immunomodulatory activity. ${ }^{9}$ However, there is no evidence of restoration of the immune function in successfully treated immunosuppressed patients. The hallmark of HPV infection is epithelial hyperplasia and retinoids have an endogenous antiproliferative effect. It has been proposed that the retinoids by altering keratinisation are able to inhibit replication and assembly of the virus, which requires keratinocytes in an advanced rate of differentiation. ${ }^{10}$ An inverse relation was observed between concentration of retinoids and HPV-DNA within infected epithelial cells, suggesting a downregulation of viral replication by the retinoids. ${ }^{11}$ In addition, their potent apoptotic activity may also play a part. ${ }^{12}$

In the present study, isotretinoin showed significantly increased efficacy compared to placebo in cervical RCA, with

\section{Key messages}

- Conventional therapies for HPV infection are often associated with unsatisfactory response rates and high recurrence rates. The use of a systemic agent may more effectively control the virus.

- This is the first double blind placebo controlled study documenting the efficacy and safety of oral retinoids in the treatment of genital warts.

- Compared to previous experience, low dose oral isotretinoin $(0.5 \mathrm{mg} / \mathrm{kg} /$ day $)$ is as effective as $1 \mathrm{mg} /$ $\mathrm{kg} /$ day isotretinoin, but it is safer and more cost effective. 
minor side effects and a relatively low recurrence rate. The efficacy was not significantly different compared to previous experience, ${ }^{78}$ although the total dose and the cost were halved. Because of its teratogenic effect, the use of isotretinoin as first line therapy for genital warts in women of reproductive age is unacceptable. Selection of patients to be treated and strict contraceptive measures are of crucial importance. In conclusion, low dose oral isotretinoin may represent an efficacious, safe, and cost effective alternative systemic therapy for cervical RCA.

\section{CONTRIBUTORS}

SG designed and organised the study; ACK analysed the results, performed the statistical analysis, and prepared the manuscript; CG and EB were responsible for clinical evaluation an follow up and recorded the data; AM performed the gynaecological examination.

\section{Authors' affiliations \\ S Georgala, A C Katoulis, C Georgala, E Bozi, A Mortakis, National and Kapodistrian University of Athens, Department of Dermatology and Venereology, "A Sygros" Hospital, Athens, Greece}

Correspondence to: Associate professor S Georgala, "A Sygros" Hospital, 5 Dragoumi Street, 16121 Athens, Greece; eykał@panafonet.gr

Accepted for publication 6 November 2003

\section{REFERENCES}

1 Garland SM. Human papillomavirus update with a particular focus on cervical disease. Pathology 2002;34:213-24.

2 Armstrong DKB, Maw RD, Dinsmore WW, et al. Combined therapy trial with interferon alpha-2a and ablative therapy in the treatment of anogenital warts. Genitourin Med 1996;72:103-7.

3 Zouboulis CC. Retinoids - which dermatological indications will benefit in the near future? Skin Pharmacol Appl Skin Physiol 2001;14:303-15.

4 Sporn MB, Roberts $A B$. Cervical dysplasia regression induced by all-transretinoic acid. J Natl Cancer Inst 1994;86:476-7.

5 Meyskens FL. Recent advances in the management of cancer with retinoids. Ann Oncol 1994;5(Suppl 9):529-32.

6 Olsen EA, Kelly FF, Vollmer RT, et al. Comparative study of systemic interferon alfa-nl and isotretinoin in the treatment of resistant condylomata acuminata. J Am Acad Dermatol 1989;20:1023-30.

7 Tsambaos D, Georgiou S, Monastirli A, et al. Treatment of condylomata acuminata with oral isotretinoin. J Urol 1997; 158:1810-2.

8 Cardamakis EK, Kotoulas IG, Dimopoulos DP, et al. Comparative study of systemic interferon alfa-2a with oral isotretinoin and oral isotretinoin alone in the treatment of recurrent condylomata acuminata. Arch Gynecol Obstet 1996;258:35-41

9 Katz RA. Isotretinoin treatment of recalcitrant warts in an immunosuppressed man. Arch Dermatol 1986;122:19-20.

10 Lutzner MA, Blanchet-Bardon C, Puissant A. Oral aromatic retinoid (RO 109359) treatment of two patients suffering with the severe form of epidermodysplasia verruciformis. In: Orfanos CE, Braun-Falco O, Farber EM, eds. Retinoids: advances in basic research and therapy. New York: SpringerVerlag, 1981:407-10.

11 Stellmach V, Leask A, Fuchs E. Retinoid-mediated transcriptional regulation of keratin genes in human epidermal and squamous cell carcinoma cells. Proc Natl Acad Sci USA 1991;88:4582-6.

12 Oridate N, Lotan D, Follen Mitchell M, et al. Inhibition of proliferation and induction of apoptosis in cervical carcinoma cells by retinoids: implications for chemoprevention. J Cell Biochem 1995;(Suppl 23):80-6.

\section{$\mathrm{ECHO}$}

\section{Intestinal B cells in AIDS}

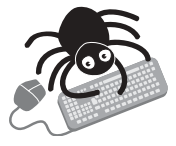

Please visit the Sexually Transmitted Infections website [www. stijournal.com] for a link to the full text of this article.
$\mathrm{T}$ here have been few studies of intestinal mucosal immunity in patients with HIV infection. Some have reported low numbers of IgA producing immunocytes in the gut mucosa but others have found normal numbers. Now researchers in Norway have demonstrated hyperactivation of intestinal B cells in patients with AIDS.

They studied 31 patients (25 men) aged 26-52 years with HIV-1 infection (25 with AIDS). Eighteen were homosexual men and eight intravenous drug users; five had acquired the infection heterosexually. Duodenal biopsy was performed on all 31 patients and specimens were examined by in situ two colour immunofluorescence staining to quantify mucosal immunoglobulin (Ig) class and subclass producing immunocytes (plasmablasts and plasma cells). Eleven healthy, HIV seronegative, age matched controls volunteered for duodenal biopsy.

The median proportions of Ig class specific duodenal immunocytes were similar in patients and controls (patients: $\operatorname{IgA} 74.6 \%, \operatorname{IgM} 19.5 \%, \operatorname{IgG} 3.4 \%$; controls: $\operatorname{IgA} 74 \%, \operatorname{IgM}$ $21 \%$, IgG 3.4\%). Among patients there was a significant increase of Ig producing cells compared with controls (175 v 120 cells per mucosal unit) due to an increase in IgA producing cells. Patients with Kaposi's sarcoma, Pneumocystis carinii pneumonia, cryptosporidiosis, or candidiasis had the greatest numbers of IgA producing cells. The proportion of IgGl immunocytes in relation to total IgG was also increased in the mucosa of patients (median $81.8 \%$ of IgG producing cells) compared with controls $(68.4 \%)$. Patients on highly active antiretroviral therapy (HAART) had fewer IgA producing cells (median 112/mucosal unit) than patients on no antiretroviral treatment (181/mucosal unit). Patients on two nucleoside analogues had intermediate numbers of IgA producing cells (124/mucosal unit).

Patients with advanced HIV-1 infection have increased numbers of IgA and IgGl producing cells in duodenal mucosa. This finding is particularly marked in patients with secondary infections. Treatment with HAART may reverse the changes. It is suggested that these findings could help in the development of mucosal AIDS vaccines.

A Gut 2004;53:487-493. 